\title{
A STOCHASTIC MODEL FOR PLANNING SWINE FACILITIES
}

\author{
Lluís M. Plà-Aragonés \\ Department of Mathematics \\ Jaume II, 73 \\ University of Lleida \\ Lleida, 25001, SPAIN
}

\begin{abstract}
A simulation model and its application for planning swine facilities are presented. Swine production is becoming more and more specialized, hence the sizing of a farm producing piglets is the main strategic decision concerning farmers who invest in sow production, since a farm comprises a big range of facilities with many possible sizes. The classical approach is deterministic, including sometimes some security margins without considering variations in future sow performance or in the management policy. The stochastic model presented here has revealed practical differences with respect to deterministic approaches. As result, simulation is useful to determine accurately the capacity, improve farm design, prevent practical problems and fit housing cost. Furthermore, the implementation in Extend allows potential users to perform efficiently different kinds of analyses.
\end{abstract}

\section{INTRODUCTION}

Recently many software programs for pig farm management have been developed and introduced for on-farm use, but they have not been widely used (Kamp 1999, Gelb 1999). For instance, there are farm management areas where decision tools are less developed, especially when strategic decisions are involved. This is a surprising fact, taking into account that strategic decisions are important for farm viability, which requires in getting the big decisions right and correctly making the major tactical adjustments as Pannell et al. (2000) noted. Such a case is the planning of swine production facilities: a typical problem when some facilities with different capacities for swine production may be built.

Classical models for designing and sizing sow farms are based on static and deterministic models. Models represent herd structure, which is compounded by number of sows in different reproductive stages. Usually, operations in sow farms are ordered by three main reproductive stages: breeding, gestation and lactation, so average time intervals associated to them are essential for planning housing facilities (Wittemore 1998). Finally, in order to account possible variations in farm management related to room availability, some security margins, more or less empirical, are applied.

Other approaches have been tested. For instance, Singh (1986) proposed a simulation model to select the optimum capacity of swine production facilities considering batch management. Later on, Lippus et al. (1996) adapted a previous Markov chain model, developed by Jalvingh et al. (1992), to support strategic planning for housing facilities for sows. They considered immediate replacement of sows, constant herd size and weekly management. It is worth pointing out that no methodological comparison was carried out in any of the cases. Thus, in this work we present an application for planning swine facilities based on a simulation model representing the occupancy of the facilities. Using a sample farm we show the differences between deterministic approaches and the stochastic one. Moreover, maximum needs of room in lactation facility, the most constraining facility in sow farms, is investigated.

\section{THE HERD MODEL}

The piglet production system is a specialization of the swine production industry characterized by a herd of sows in a continuous process of reproduction. The piglets are the commercial product, which after weaning are sold to rearing-fattening farms. This specialization gives additional efficiency gains as Rowland et al. (1998) pointed out and it is widely extended within the Spanish swine industry.

Normally, confinement facilities consist of a service facility, a gestation facility and a farrowing facility with multiple farrowing rooms. The farrowing facility is divided in multiple rooms for better disease and parasite control. All facilities may be housed in one or in several buildings. The service facility houses breeding sows, gilts (young sows) and boars. The weaning of all litters from a farrowing room, i.e. a batch, is practiced to synchronize breeding and farrowing in the next cycle. Uniformly during the re- 


\section{Plà-Aragonés}

productive cycle sows are culled for different reasons, and the size of batches are completed after weaning in the service facility with replacement gilts. Culled sows can remain on the farm until they are sent to the slaughterhouse. Culled or dead sows can be replaced immediately, but usually after some farm specific delay. Replacement gilts and sows are generally kept in the service facility to be inseminated. They are moved from service to gestation facility when pregnancy is confirmed, if not, they remain to be reinseminated. Gilts and sows in the gestation facility belonging the same batch are moved into the farrowing room approximately once per week before parturition. To synchronize the breeding and farrowing of a group of sows, all litters from a farrowing room are weaned simultaneously and are sent to the nursery or sold. The sows, after weaning, are sent back to the service facility. The farrowing room is cleaned, sterilized and closed for a drying period. After the drying period, the room is ready to receive the next batch of sows.

\subsection{Model Formulation}

The sow herd model used in this work has been adapted from a semi-Markov decision model presented by Plà et al. (2004). In that model, sows move from one state to another through transitions. The embedded process is represented as a Markovian decision model defined by the following elements: states $S$ in which sows can be observed (usually, these states are related to reproduction states), actions $A$ that farmer can take at each stage, transition probabilities for sows evolving from one state to another and the reward function representing the profit (positive or negative) associated to each transition. States and actions are finite sets. In a infinite planning horizon, $\Omega=\{\mathrm{S} \times \mathrm{A}\}^{\infty}$ represents the set of all possible system paths $\omega$, i.e. all possible sequences of states and actions, $\omega=\left(i_{1}, a_{1}, i_{2}, a_{2}, \ldots, i_{\mathrm{n}}, a_{\mathrm{n}}\right.$ $, \ldots) \in \Omega$. The sequence of actions is the result of a policy or strategy $D$.

For each stationary policy, $D, P^{D}=\left(p_{i j}^{D}\right)$ is the transition matrix representing transitions of sows from one state $i \in S$ to another $j \in S$ when policy $D$ is adopted by the farmer. The time period between states is coincident with the time period between actions and it is called stage. Future states $\mathrm{S}$ are defined as being only conditioned by the present state and not by the manner to which the present state is reached (Markovian property).

The reward function, $r$, represents the farmer's preferences in a decision theory context and it can be used in the design of a performance criterion, like the total return expected after $\mathrm{n}$ transitions from the initial state $i$, as follows:

$$
B_{n}^{D}(i)=r_{i}^{D}+\sum_{j \in S} p_{i j}^{D} B_{n-1}^{D}(j)
$$

where the reward function $r_{i}^{D}$ is the expected net return from a sow in the $i$-state when policy $D$ is adopted. Therefore, the dynamics of a herd under any given policy can be represented as a finite irreducible and aperiodic Markov chain. The expected distribution at equilibrium, $\Pi P=\left(\pi_{1}^{D}\right.$, $\left.\pi_{2}^{D}, \ldots, \pi_{|S|}^{D}\right)$, is calculated solving the following linear system of equations,

$$
\begin{aligned}
& \pi_{j}^{D}=\sum_{k \in S} \pi_{k}^{D} p_{k j}^{D} \quad j \in S \\
& \sum_{j \in S} \pi_{j}^{D}=1
\end{aligned}
$$

where: $p_{k j}^{D}$ represents the transition probability for a sow to pass from state $j$ to state $k . D$ is the management strategy of the farmer and $\left\{\pi_{j 1}^{D}, \mathrm{j} \in S\right\}$ represents the distribution at equilibrium of the embedded process when policy $D$ is adopted by the farmer.

For our purpose, several changes in the above model formulation need to be introduced. Because the farmer's policy is unique and stable for a long period of time in accordance with the stationarity assumption for policies, the transition matrix is unique, once the deterministic policy has been set (then, from now on the symbols of policy and actions will be dropped in the notation). Furthermore transitions to represent movements among facilities have to be introduced and therefore the state set is redefined, thus, transitions can represent the movement of sows between facilities or sows changing reproductive state. Moreover, stages are intervals of different times, depending on the current state of the sows and therefore a new group of parameters have to be added. The average time between transitions, $\tau_{i}$, is the expected time in days that sows spend in state $i, i \in S$. As result the model becomes a semi-Markov chain where steady-state distribution can be easily calculated. Calculations can be performed in two steps; the first one using (1), and the second one accounting for the average time between transitions:

$$
\pi_{j}^{*}=\pi_{j} \tau_{\mathrm{j}} \quad j \in S
$$

Final limit herd distribution over states, $\left\{\pi_{j}^{*}, j \in \mathrm{S}\right\}$, is obtained after normalizing the vector obtained in (2). Herd distribution is used to estimate the capacity of different facilities. To do so, the state set has to be partitioned into three, related to service (or insemination), gestation and farrowing-lactation facilities (i.e. $S=I \cup G \cup L$, respectively). Assigning each original state to its facility, we obtain: 


$$
H_{F}=\frac{\sum_{j \in F} \pi_{j}^{*}}{\sum_{j \in S} \pi_{j}^{*}} F \in\{I, G, L\},
$$

where $H_{F}$ is the distribution of occupation over facility $F$, and $\left\{\pi_{j}^{*}, j \in S\right\}$ has been calculated using (2).

The previous model as described provides the pattern from which the simulation model was developed. The simulation model is based on the aggregation of individual sow performances simulated over time. Each sow is represented individually and owing their specific parameters sampled from the general parameters of the farm. Hence, additional parameters and productive thresholds are introduced into the original semi-Markov model to better represent usual reproductive management in actual sow farms.

\subsection{Model Implementation}

The simulation model was implemented in Extend, an interactive simulation tool (Krahl 2003).

The Extend simulation environment provides the tools for all levels of modelers to create accurate, credible, and usable models in an efficient way. Extend was chosen because it facilitates every phase of the simulation project, from creating, verifying, and validating the model, to the construction of a user interface which allows others to analyze the system. An additional advantage for developers is the Extend's built-in, compiled language, ModL, to create reusable modeling components. All of this is done within a single, self-contained software program that does not require external interfaces, compilers, or code generators.

Model structure is presented in Figure 1. Main elements of the model are: the block of general parameters, the block representing the farm and the Display Simulation block. All of them are tailored made blocks that can be complemented with standard ones as the Plotter I/O block.

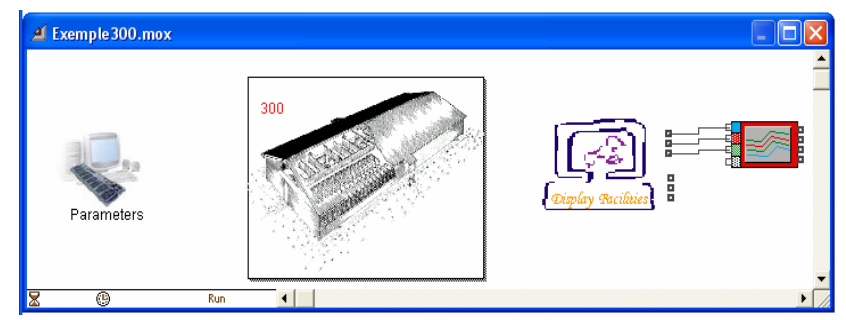

Figure 1: General Model Structure

The block of general parameters and this of the farm are hierarchical blocks, that is, they are built from other blocks. For instance, the block of parameters contains five blocks each one representing a set of parameters affecting herd dynamics: casualties, conception, abortion, economic issues and technical issues.
The block of the farm contains blocks representing each individual sows (Figure 2), one for each animal that can be housed in. For an easier handling, sows can be grouped in batches of different size.

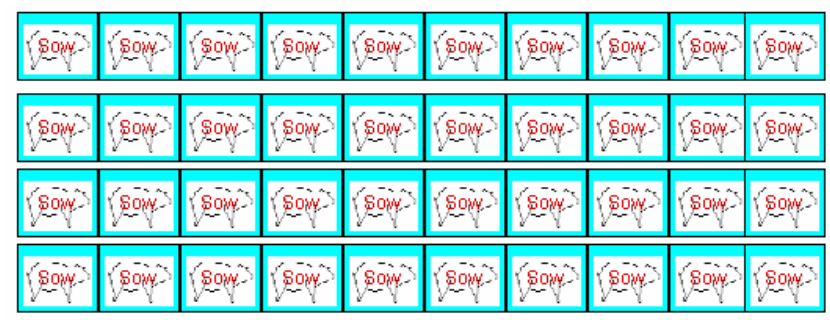

Figure 2: Contents of the Farm Model

The Display Simulation block is used to determine the structure of the farm in three or two facilities and to display the structure over time in combination with the Plotter I/O block.

\subsection{Model Verification}

According to Kleijnen (1995), the model was verified comparing final simulation outputs with analytical results. From (1) and (2) herd structure at equilibrium can be derived and compared with simulation outputs. For this purpose the complete version of the simulation model was used, but selecting only those compatible parameters detailed in Appendix A. In Table 1 sow herd distribution over main states is presented while in Table 2 it is presented by reproductive cycle.

Table 1: Herd Distribution over Main States (Herd Size: 300, Time Simulated: 10000 Days)

\begin{tabular}{|l|l|l|l|l|}
\hline Facility & \multicolumn{3}{|c|}{ Simulated result } & Analytical \\
& $\mathrm{t}=1000$ & $\mathrm{t}=5000$ & $\mathrm{t}=10000$ & result \\
\hline Open & 45 & 22 & 32 & 32 \\
\hline Gestation & 209 & 257 & 237 & 237 \\
\hline Lactation & 46 & 21 & 31 & 31 \\
\hline
\end{tabular}

Given herd structure as a distribution, the chi-square test was used to measure if the observed steady-state is equal to the known (analytical) herd structure at equilibrium. Convergence was also analyzed in this way to study initialization bias (results not shown).

Table 2: Herd Distribution by Reproductive Cycle (Herd Size:300, Time Simulated: 10000 Days)

\begin{tabular}{|l|l|l|l|l|}
\hline Cycle & \multicolumn{3}{|c|}{ Simulated result } & Analytical \\
& $\mathrm{t}=1000$ & $\mathrm{t}=5000$ & $\mathrm{t}=10000$ & result \\
\hline 1 & 212 & 208 & 206 & 206 \\
\hline 2 & 63 & 57 & 60 & 64 \\
\hline 3 & 20 & 26 & 22 & 20 \\
\hline 4 & 3 & 9 & 10 & 6 \\
\hline 5 \&more & 2 & 0 & 2 & 4 \\
\hline
\end{tabular}




\section{CASE STUDIES}

In order to assess the suitability and to measure possible advantages of the simulation model proposed in planning swine facilities, a comparison was established between this new stochastic approach and deterministic ones through a sample farm. Thus, a group of parameters satisfying the needs of both methodologies was set. These parameters represent the management policy related to facilities and production level that the farmer wants to implement or achieve. The data on biological production parameters used in this paper were inspired from computerized records of Bd-Porc databank (2000), the main Spanish pig information system and from literature. All parameters used in this case example and described below are presented in Appen$\operatorname{dix} B$.

\subsection{Case Parameters}

Nowadays, we can consider about $600-700$ sows as a reasonable size of a sow farm producing piglets in Spanish conditions. For the example, the number of sows were fixed at 660. Facilities involved were service, gestation and lactation. All gilts were purchased from outside and moved into the service facility waiting the first insemination two weeks after; home-grown sows are not represented in the model, since they are rare in commercial Spanish conditions. The sojourn time in service facility was fixed at a minimum of 21 days post-fertile insemination. Overall occupancy would range from 35 to 39 days, after which sows were moved to gestation dependencies. One week before farrowing, sows were moved to farrowing dependencies; as a result they had been in the gestation facility for 79 days (assuming gestations of 114 days). Lactation was fixed at 21 days, so that the total time interval in this facility was 35 days, including a drying period of one week. Finally, after weaning, sows re-entered service dependencies and batches were completed when needed. Culled sows were not immediately sent to the slaughterhouse, a time period depending on the last state visited before culling was considered. The nutritional effects on reproduction are taken into account through the reproductive parameters.

The model assumed 11 cycles as the maximum number of reproductive cycles. The average of time intervals for each reproductive state was considered; these parameters are assumed to be independent of the reproduction cycle.

Marginal probabilities for conception rates can be affected by the number of unsuccessful inseminations and by the reproduction cycle. Conception rates are expressed in relation to all matings per cycle. Abortion marginal probabilities are also specific for each reproduction cycle, whereas culling marginal probabilities are specific for each reproduction cycle and state. Only conception rates for first, second and third inseminations are given because it is very unusual to find farms with more than two inseminations per cycle. A culling rate of $100 \%$ in the last cycle and state has been established as ending of the sow lifespan.

\subsection{Results from Deterministic Models}

Firstly, using the parameters presented in the previous section, the herd structure in the steady-state was derived from (2) and (3). A second model based on the classical method used by engineers in sow farm sizing for different facilities (service, gestation, farrowing-lactation) was also solved. It assumes a fixed time interval per facility and a homogeneous rate of occupancy and the procedure is described in more detail in Brent (1986) and Wittemore (1998). Although all of the parameters are customizable, the simplest version of them was considered for illustrative purpose and easier comparison between models (e.g. the effect of cycle and number of insemination on different transitions are ignored as shown in Appendix B). Both models considered an immediate replacement of culled animals (constant herd size over time). In Table 2, we show the results obtained for each model.

\subsection{Results of the Simulation Model}

The whole set of parameters are used to run the simulation. A first run was made just to compare the output with previous models. A period of 10000 days was simulated and the last 1000 days were used to make calculations. Results are presented in Table 3.

Table 3: Number of Stalls Required per Facility

\begin{tabular}{|l|c|c|c|}
\hline Facility & $1^{\text {st }}$ model & $2^{\text {nd }}$ model & Simulation \\
\hline Service & 223 & 163 & 192 \\
\hline Gestation & 341 & 367 & 357 \\
\hline Lactation & 121 & 163 & 137 \\
\hline
\end{tabular}

\subsection{Further Results}

After modifying different settings and management practices, a second run is performed. In this second run a more realistic situation is analyzed and the stress is put on maximum and minimum margins for lactation facility at steady-state.

As Figure 3 shows, during daily operation slight variations might appear concerning room needs in sow farms. These variability can easily managed in Service and Gestation facilities. More problematic is to manage the possible overflow of the lactation facility. Lactation facility is the more constraining facility in a sow farm. Hence, the determination of the average capacity is not enough, variability becomes also important in scheduling daily operations on farm. 


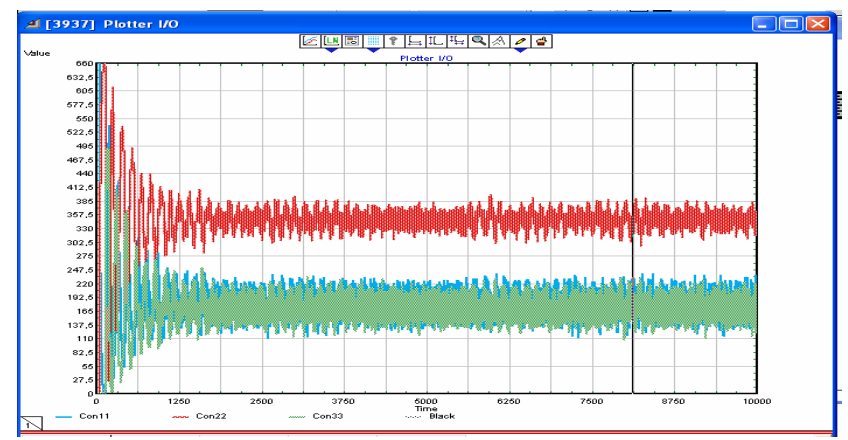

Figure 3: Plotter of the Simulation Output

With this purpose, and taking the same set of parameters presented in Appendix B the variability associated to this facility was investigated. In particular, expected maximum and minimum room needs are estimated and confidence intervals of $95 \%$ calculated from running a long simulation. Results assuming i.i.d. data are presented in Table 4.

Table 4: Farrowing Crates in Lactation Facility

\begin{tabular}{|c|c|c|}
\hline $\begin{array}{l}\text { Average } \\
(\mathrm{n}=10000)\end{array}$ & $\begin{array}{l}\text { Maximum } \\
(\mathrm{n}=1162)\end{array}$ & $\begin{array}{l}\text { Minimum } \\
(\mathrm{n}=1047)\end{array}$ \\
\hline 160 & $174.5 \pm 2.1$ & $147.1 \pm 1.5$ \\
\hline
\end{tabular}

\section{DISCUSSION}

The simulation model presented here for planning swine facilities in sow farms has methodological advantages over other approaches. For instance the graphical interface, animation capabilities and connectivity with other applications as spreadcheets represent an improvement respect the model proposed by Singh (1986). On the other hand, the model presented by Lippus et al. (1996) was deterministic, based on a Markov model rather similar to the first model presented here, but Lippus et al. when considering weekly transitions introduced an artificial augmentation of the state set that made the model complex. Such a complexity was unnecessary because they were concerned in steadystate distribution of sows over facilities that can be calculated directly from (2) and (3) as it is shown here.

Model verification demonstrated that the simulation model performed well when calculating sow herd distribution over time as shown in Table 1 and 2. However, it is observed how always there is present a variation in the number of stall occupied over time. This variation is present around a mean value that is stable and consistent with deterministic calculations.

The first case study served to compare two common approaches in a more real situation to the simulation model. Table 3 showed interesting differences between approaches. The second model is the simplest to calculate and the worst at the same time. It is a static and deterministic model that underestimates Service facility needs and overestimates those in Lactation facilities. The first model should be similar to simulation outcomes, but differences appears because, for instance, simulation is more flexible to represent casualties in farm as well as other exceptions difficult to be handled under a semi-Markov framework. Then the first model penalize Lactation in favor of Service facility. Simulation results seems to represent better actual farm management and fit better housing facilities for sows.

The second case study focused on variability affecting Lactation facilities due to regular herd dynamics. In some cases, these situations can introduce instability to the herd and problems in the scheduling of daily operations. Thus, the results of a long run simulation presented in Table 4 showed how the average size for the lactation facility is of 160 farrowing crates, but in daily operation, needs of 174.4 in average are registered. These figures could be very interesting for practical use in planning swine facilities and avoiding the overflow of Lactation facilities.

Finally, calculations concerning other different management strategies are easy to perform. The simulation herd model is simple and accounts for their own library as a repository for different blocks that can be used to accomplish different tasks.

\section{CONCLUSIONS}

The simulation model described here represents a practical approach for planning swine facilities under different farm management strategies. It is more flexible and accurate than deterministic approaches, essentially because it better captures the dynamics of the sow production and reproduction process. Moreover, different advantages are drawn respect to previously published models for the same purpose. Deterministic methods are based on the average time interval for each reproductive stage which is considered as a part of the reproductive management policy adopted by the farmer. The simulation model considers variations in sow performance, and can be adapted to possible variations in the management policy, in order to explore alternative optimal capacity of facilities. We have shown how the Lactation facility size is in general bad estimated by deterministic methods and how daily variations in room needs are important. The model is an easy-to-use tool, useful to gain insight into the occupancy rate of swine facilities. As a result, housing needs can be better fitted.

\section{APPENDIX A: VERIFICATION PARAMETERS}

The parameters enumerated here are classified in time intervals (Table A-1), transition probabilities (Table A-2). The maximum lifespan considered was 11 reproductive cycles and a maximum of two matings. The farm size was set to 300 sows. No abortions were considered and time intervals were constant. For verification parameters were not affected by reproductive cycle. 
Table A-1: Time Intervals

\begin{tabular}{|l|c|}
\hline Time interval & days \\
\hline Interval to mating & 7 \\
\hline Interval between matings & 21 \\
\hline Gestation & 114 \\
\hline Lactation & 21 \\
\hline Interval mating to culling & 0 \\
\hline Interval otherwise to culling & 0 \\
\hline $\begin{array}{l}\text { Time interval to be moved to ges- } \\
\text { tation facility after mating }\end{array}$ & 28 \\
\hline $\begin{array}{l}\text { Time interval to be moved to lac- } \\
\text { tation facility before farrowing }\end{array}$ & 7 \\
\hline Drying period after weaning & 7 \\
\hline
\end{tabular}

Table A-2: Transition Probabilities

\begin{tabular}{|l|c|}
\hline & Probability \\
\hline Conception & 0.80 \\
\hline Mortality & 0.08 \\
\hline Casualties & 0.12 \\
\hline
\end{tabular}

\section{APPENDIX B: CASE EXAMPLE PARAMETERS}

The parameters enumerated here, as above, are classified in time intervals (Table B-1), transition probabilities (Table 8) and management requirements. Distribution of time intervals shown in Table B-1 are extracted from the exploratory analysis introduced in Marin et al. (2005). Management requirements are the maximum lifespan, fixed at 11 reproductive cycles and the maximum number of mating per reproductive cycle (two matings maximum). Furthermore, only two abortions per sow are allowed and weanings are scheduled every three weeks, all litters with more than two weeks of lactation are weaned. The farm size was set to 660 sows.

Table B-1: Time Intervals

\begin{tabular}{|l|c|}
\hline Time interval & $\begin{array}{l}\text { Distribution } \\
\text { in days }\end{array}$ \\
\hline Interval to mating & $\Gamma(7,0.5)$ \\
\hline Interval between matings & $\mathrm{N}(21,3)$ \\
\hline Gestation & $\mathrm{N}(115,2)$ \\
\hline Abortion & weib $(5,80)$ \\
\hline Lactation & 21 \\
\hline Interval mating to culling & $\mathrm{N}(28,3)$ \\
\hline Interval otherwise to culling & $\mathrm{N}(7,3)$ \\
\hline $\begin{array}{l}\text { Time interval to be moved to ges- } \\
\text { tation facility after mating }\end{array}$ & 28 \\
\hline $\begin{array}{l}\text { Time interval to be moved to lac- } \\
\text { tation facility before farrowing }\end{array}$ & 7 \\
\hline Drying period after weaning & 7 \\
\hline
\end{tabular}

Table 8: Transition Probabilities

\begin{tabular}{|l|c|}
\hline & Probability \\
\hline Conception & 0.80 \\
\hline Abortion & 0.02 \\
\hline Mortality & 0.08 \\
\hline Casualties & 0.12 \\
\hline
\end{tabular}

\section{ACKNOWLEDGMENTS}

This work has been done by funding of Spanish Ministry of Science and Education, project code: AGL2002-03960. The author would like acknowledge the advice and support of Dr. Cándido Pomar Dr. Jesús Pomar and Dr. Javier Faulín in different stages of the development of this work.

\section{REFERENCES}

Bd-Porc. 2000. Bd-Porc Database. Available via <http: / /bdporc.irta.es/index.j.sp> [accessed March 4, 2005]

Brent, G. 1986. Housing the pig. Farming Press.

Gelb, E.M. 1999. Adoption of IT by farmers- Does reality reflect the potential benefit?. In Proceedings Second European Conference EFITA, 433-441. Bonn, Germany.

Jalvingh, A.W., A.A. Dijkhuizen and J.A.M. van Arendonk. 1992. Dynamic probabilistic modelling of reproduction and management in sow herds. General aspects and model description. Agricultural Systems 39: 133-152.

Kamp, J.A.L.M. 1999. Knowledge based systems: from research to practical application. Pitfalls and critical success factors. Computers and Electronics in Agriculture 22: 243-250.

Kleijnen, J.H.P. 1995. Verification and validation of simulation models. European Journal of Operational Research 82: 145-162.

Krahl, D. 2003. Extend: an interactive simulation tool. In Proceedings of the 2003 Winter Simulation Conference, ed. S. Chick, P. J. Sánchez, D. Ferrin, and D. J. Morrice, 198-196. IEEE, Piscataway, NJ.

Lippus, A.C., A.W. Jalvingh, J.H.M. Metz and R.B.M. Huirne. 1996. A dynamic probabilistic model for planning housing facilities for sows. Transactions of the ASAE 39: 1215-1223.

Marín, J.M., L.M. Plà and D. Ríos. 2005. Inference for some stochastic process models related with sow farm management. Journal of Applied Statistics. In Press.

Panell, D.J., B. Malcom and R.S. Kingwell. 2000. Are we risking too much? Perspectives on risk in farm modelling. Agricultural Economics 23: 69-78.

Plà, L.M., D. Babot and J. Pomar. 2004. A mathematical model for designing and sizing sow farms. International Transactions in Operational Research. 11: 485494. 
Rowland, W.W., M.R. Langemeier, B.W. Schurle and A.M. Featherstone. 1998. A nonparametric efficiency analysis for a sample of Kansas swine operations. Journal of Agricultural and Applied Economics. 30:189-199.

Singh, D. 1986. Simulation-aided capacity selection of confinement facilities for swine production. Transactions of the ASAE 29, 807-815.

Wittemore, C. 1998. The science and practice of pig production. Oxford. Blackwell Science Ltd.

\section{AUTHOR BIOGRAPHY}

LLUIS M. PLA is an associate professor in the Department of Mathematics at University of Lleida (UdL) and a Senior Researcher in the Area de Producción Animal at the UdL-IRTA Center. His research interests include operational research methods applied in agriculture and forest management, with special reference to simulation, dynamic programming, planning, Markov decision processes and production planning. Coordinator of the EURO-Working group: Operational Research in Agriculture and Forest management. He is a member of INFORMS and EURO. His e-mail address is $<$ lmpla@matematica.udl.es> and his Web address is <http://www. udl. es/usuaris/d4089492/>. 